\title{
Generalized Higgs inflation
}

\author{
Kohei Kamada, ${ }^{1, \dagger}$ Tsutomu Kobayashi, ${ }^{2,3, *, \$}$ Tomo Takahashi, ${ }^{4, \S}$ Masahide Yamaguchi, ${ }^{5, \|}$ and Jun'ichi Yokoyama ${ }^{6,7, \mathbb{I}}$ \\ ${ }^{1}$ Deutsches Elektronen-Synchrotron DESY, Notkestrasse 85, D-22607 Hamburg, Germany \\ ${ }^{2}$ Hakubi Center, Kyoto University, Kyoto 606-8302, Japan \\ ${ }^{3}$ Department of Physics, Kyoto University, Kyoto 606-8502, Japan \\ ${ }^{4}$ Department of Physics, Saga University, Saga 840-8502, Japan \\ ${ }^{5}$ Department of Physics, Tokyo Institute of Technology, Tokyo 152-8551, Japan \\ ${ }^{6}$ Research Center for the Early Universe (RESCEU), Graduate School of Science. The University of Tokyo, \\ Tokyo 113-0033, Japan \\ ${ }^{7}$ Kavli Institute for the Physics and Mathematics of the Universe (IPMU), The University of Tokyo, \\ Kashiwa, Chiba, 277-8568, Japan
}

(Received 3 April 2012; published 3 July 2012)

\begin{abstract}
We study Higgs inflation in the context of generalized $G$-inflation, i.e., the most general single-field inflation model with second-order field equations. The four variants of Higgs inflation proposed so far in the literature can be accommodated at one time in our framework. We also propose yet another class of Higgs inflation, the running Einstein inflation model, that can naturally arise from the generalized $G$-inflation framework. As a result, five Higgs inflation models in all should be discussed on an equal footing. Concise formulas for primordial fluctuations in these generalized Higgs inflation models are provided, which will be helpful to determine which model is favored from the future experiments and observations such as the Large Hadron Collider and the Planck satellite.
\end{abstract}

DOI: 10.1103/PhysRevD.86.023504

PACS numbers: $98.80 . \mathrm{Cq}$

\section{INTRODUCTION}

The Higgs particle is the only undiscovered ingredient of the standard model (SM) of particle physics; it plays the fundamental role of accounting for the origin of the masses of all the known massive particles. Though some signals have been hinted at in the LHC experiments recently [1,2], we await its final discovery. The discovery of the Higgs particle would have profound implications not only in particle physics but also in cosmology, since all of the inflation models rely on the existence of a scalar field, the inflaton, driven either by its potential energy [3,4] or kinetic energy $[5,6]$. Note that even higher-curvature theories of inflation without any scalar field [7,8] may be conformally transformed to Einstein gravity with a scalar field driving inflation.

There may even be a direct connection between the Higgs field and cosmic inflation, namely, the possibility that the Higgs field itself acts as the inflaton. In order to suppress the amplitude of the curvature perturbation from the inflaton's quantum fluctuations [9], its self-coupling $\lambda$ must be smaller than $\sim 10^{-13}$ [10], which is not the case in the SM Higgs field. Hence, some extension is necessary in either gravitational or kinetic sectors of the theory.

\footnotetext{
*Present address: Department of Physics, Rikkyo University, Toshima, Tokyo 171-8501, Japan.

${ }^{\dagger}$ kohei.kamada@desy.de

${ }^{\ddagger}$ tsutomu@tap.scphys.kyoto-u.ac.jp

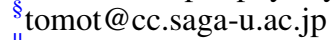

"gucci@phys.titech.ac.jp

tyokoyama@resceu.s.u-tokyo.ac.jp
}

So far, four variants of Higgs inflation have been proposed in this direction. ${ }^{1}$ The first one is to introduce a large and negative nonminimal coupling between the scalar field and the scalar curvature [12-14]. In this model, the Planck scale takes effectively a much larger value during inflation than it is today to suppress the amplitude of curvature perturbations.

The second is the new Higgs inflation model [15] whose kinetic term is coupled to the Einstein tensor [16]. This coupling changes the normalization of the field during inflation, which suppresses quantum fluctuations. The third one is running kinetic inflation [17], in which a nonstandard kinetic term simply changes the normalization of the inflaton in some domain of the field space, leading essentially to the same effect as in the previous example. Finally, the fourth model is Higgs $G$-inflation [18], where the lowest nontrivial-order Galileon-like interaction [19,20] is incorporated into the original action. Although this model contains higher-derivative interactions, the field equations remain of second order and the newly introduced term acts as an extra friction, which effectively smoothens the potential to suppress curvature fluctuations down to the observed value.

In fact, each of the above four models falls into a subclass of generalized $G$-inflation [21], which is the most general single-field inflation model having secondorder gravitational and scalar-field equations. Hence, a

\footnotetext{
${ }^{1}$ Inflationary models in which the Higgs field in supersymmetric standard models is identified as the inflaton are discussed in Ref. [11].
} 
unified treatment of apparently different Higgs inflation models is possible in the context of generalized $G$-inflation. As a by-product of this fact, we propose yet another class of successful Higgs inflation.

In this paper, we first clarify why five different Higgs inflation models exist in the context of generalized $G$-inflation. Then, we discuss their dynamics and primordial fluctuations in a unified way. In particular, the formulas of primordial fluctuations in these generalized Higgs inflation models are given in terms of the slow-roll parameters and field-dependent functions in the Lagrangian, which will be helpful to single out the model favored by the future experimental and observational data from the LHC experiment and the Planck satellite, etc. Note, however, that in the context of generalized $G$-inflation, one may well find the best-fit model in some combinations of two or more models among the five mentioned above. Indeed, the strength of the generalized $G$-inflation is that, in performing the parameter search using the Markov chain Monte Carlo (MCMC) method, all the variants of Higgs inflation models can be analyzed simultaneously and seamlessly unlike in [22].

The purpose of the present paper is therefore to clarify first how the previously known four Higgs inflation models are realized as part of the generalized $G$-inflation model and then propose the fifth model in the same context together with the formulas for curvature perturbations and tensor perturbations, as well as the non-Gaussianity of the former, which turns out to be small, in a unified manner. Note that our framework is not confined only to inflation driven by the SM Higgs field but is applicable to more general potential-driven single-field inflation models, too.

The rest of the paper is organized as follows. In Sec. II we introduce variants of Higgs inflation models in the context of generalized $G$-inflation. In Sec. III evolution of the homogeneous background and conditions for inflation are summarized. Then we calculate spectra of perturbations in Sec. IV in a unified manner. Finally, Sec. V is devoted to a discussion and conclusions.

\section{HIGGS INFLATION MODELS AS VARIANTS OF GENERALIZED $G$-INFLATION}

The tree-level SM Higgs Lagrangian is

$$
S_{0}=\int d^{4} x \sqrt{-g}\left[\frac{M_{\mathrm{Pl}}^{2}}{2} R-\left|D_{\mu} \mathcal{H}\right|^{2}-\lambda\left(|\mathcal{H}|^{2}-v^{2}\right)^{2}\right],
$$

where $M_{\mathrm{Pl}}$ is the reduced Planck mass, $D_{\mu}$ is the covariant derivative with respect to the SM gauge symmetry, $\mathcal{H}$ is the SM Higgs boson, $v$ is its vacuum expectation value, and $\lambda$ is the self-coupling constant. Taking the gauge ${ }^{t} \mathcal{H}=(0, v+\phi) / \sqrt{2}$, with $\phi$ being a real scalar field and assuming $\phi \gg v$, the action is simplified to

$$
S_{0}=\int d^{4} x \sqrt{-g}\left[\frac{M_{\mathrm{Pl}}^{2}}{2} R-\frac{1}{2}\left(\partial_{\mu} \phi\right)^{2}-\frac{\lambda}{4} \phi^{4}\right],
$$

which is nothing but the action for original chaotic inflation [4].

This model cannot serve as a viable inflation model as it is. Since the self-coupling is related with the Higgs mass $m_{H}$ as

$$
m_{H}=\sqrt{2 \lambda} v, \quad v=246 \mathrm{GeV}
$$

at the tree level, $\lambda$ cannot take a tiny value to give the correct amplitude for density fluctuations with the value indicated by the LEP collider $m_{H}>114.4 \mathrm{GeV}$ at the 95\% CL [23].

As mentioned in the Introduction, four remedies have been proposed so far, all of which can be unified as a subclass of the generalized $G$-inflation [21] whose action is given by

$$
S=\sum_{i=2}^{5} \int d^{4} x \sqrt{-g} \mathcal{L}_{i}
$$

where

$$
\begin{gathered}
\mathcal{L}_{2}=K(\phi, X), \\
\mathcal{L}_{3}=-G_{3}(\phi, X) \square \phi, \\
\mathcal{L}_{4}=G_{4}(\phi, X) R+G_{4 X}\left[(\square \phi)^{2}-\left(\nabla_{\mu} \nabla_{\nu} \phi\right)^{2}\right], \\
\mathcal{L}_{5}=G_{5}(\phi, X) G_{\mu \nu} \nabla^{\mu} \nabla^{\nu} \phi-\frac{1}{6} G_{5 X}\left[(\square \phi)^{3}-3(\square \phi)\right. \\
\left.\times\left(\nabla_{\mu} \nabla_{\nu} \phi\right)^{2}+2\left(\nabla_{\mu} \nabla_{\nu} \phi\right)^{3}\right],
\end{gathered}
$$

where $R$ is the Ricci tensor, $G_{\mu \nu}$ is the Einstein tensor, $X=-(1 / 2) g^{\mu \nu} \nabla_{\mu} \phi \nabla_{\nu} \phi,\left(\nabla_{\mu} \nabla_{\nu} \phi\right)^{2}=\nabla_{\mu} \nabla_{\nu} \phi \nabla^{\mu} \nabla^{\nu} \phi$, $\left(\nabla_{\mu} \nabla_{\nu} \phi\right)^{3}=\nabla_{\mu} \nabla_{\nu} \phi \nabla^{\nu} \nabla^{\lambda} \phi \nabla_{\lambda} \nabla^{\mu} \phi$, and $\quad G_{i X}=$ $\partial G_{i} / \partial X$. This theory was originally discovered by Horndeski [24] in a different form, and rediscovered by Deffayet et al. [25] in the present form, whose equivalence to the original theory was first shown in [21].

For a homogeneous and isotropic cosmological background, $d s^{2}=-d t^{2}+a^{2}(t) d \mathbf{x}^{2}, \phi=\phi(t)$, the $(t t)$ component of the gravitational field equations reads

$$
\sum_{i=2}^{5} \mathcal{E}_{i}=0
$$

where

$$
\begin{gathered}
\mathcal{E}_{2}=2 X K_{X}-K, \\
\mathcal{E}_{3}=6 X \dot{\phi} H G_{3 X}-2 X G_{3 \phi}, \\
\mathcal{E}_{4}=-6 H^{2} G_{4}+24 H^{2} X\left(G_{4 X}+X G_{4 X X}\right) \\
-12 H X \dot{\phi} G_{4 \phi X}-6 H \dot{\phi} G_{4 \phi},
\end{gathered}
$$




$$
\begin{aligned}
\mathcal{E}_{5}= & 2 H^{3} X \dot{\phi}\left(5 G_{5 X}+2 X G_{5 X X}\right)-6 H^{2} X\left(3 G_{5 \phi}\right. \\
& \left.+2 X G_{5 \phi X}\right),
\end{aligned}
$$

with $H=\dot{a} / a=d \ln a / d t$. This corresponds to the Friedmann equation, which can be easily verified by substituting $G_{4}=M_{\mathrm{Pl}}^{2} / 2=\mathrm{const}$ and $G_{3}=0=G_{5}$ into the above equations. The scalar-field equation of motion is given by

$$
\frac{1}{a^{3}} \frac{d}{d t}\left(a^{3} J\right)=P_{\phi}
$$

where

$$
\begin{aligned}
J= & \dot{\phi} K_{X}+6 H X G_{3 X}-2 \dot{\phi} G_{3 \phi}+6 H^{2} \dot{\phi}\left(G_{4 X}\right. \\
& \left.+2 X G_{4 X X}\right)-12 H X G_{4 \phi X}+2 H^{3} X\left(3 G_{5 X}\right. \\
& \left.+2 X G_{5 X X}\right)-6 H^{2} \dot{\phi}\left(G_{5 \phi}+X G_{5 \phi X}\right)
\end{aligned}
$$

and

$$
\begin{aligned}
P_{\phi}= & K_{\phi}-2 X\left(G_{3 \phi \phi}+\ddot{\phi} G_{3 \phi X}\right)+6\left(2 H^{2}+\dot{H}\right) G_{4 \phi} \\
& +6 H(\dot{X}+2 H X) G_{4 \phi X}-6 H^{2} X G_{5 \phi \phi} \\
& +2 H^{3} X \dot{\phi} G_{5 \phi X} .
\end{aligned}
$$

The space-space component of the gravitational field equations is not independent of the generalized Friedmann and scalar-field equations.

Although the generalized $G$-inflation covers all the possible single-field inflation models including the ones driven by $\phi$ 's kinetic energy, since we are interested in potentialdriven inflation here, we focus on its subclass for which each function in the Lagrangian can be Taylor expanded in terms of $X$ as

$$
\begin{gathered}
K(\phi, X)=-V(\phi)+\mathcal{K}(\phi) X+\cdots, \\
G_{i}(\phi, X)=g_{i}(\phi)+h_{i}(\phi) X+\cdots
\end{gathered}
$$

Hereafter, we will neglect all the higher order terms in $X$. Using this Taylor-expanded form, one can handle a vast class of potential-driven inflation models while avoiding the situation where the equations are too general to tell anything concrete.

We note here the following identities:

$$
\begin{gathered}
g_{3}(\phi) \square \phi=2 g_{3}^{\prime} X+(\text { t.d. }), \\
g_{5}(\phi) G^{\mu \nu} \nabla_{\mu} \nabla_{\nu} \phi=-g_{5}^{\prime}\left[X R+(\square \phi)^{2}-\left(\nabla_{\mu} \nabla_{\nu} \phi\right)^{2}\right] \\
+3 g_{5}^{\prime \prime} X \square \phi-2 g_{5}^{\prime \prime \prime} X^{2}+\text { (t.d.), }
\end{gathered}
$$

where a prime denotes differentiation with respect to $\phi$ and (t.d.) represents total derivative terms. These identities allow us to set $g_{3}=0=g_{5}$ without loss of generality. In particular, the derivative coupling to the Einstein tensor in new Higgs inflation, $G^{\mu \nu} \partial_{\mu} \phi \partial_{\nu} \phi$, is obtained most straightforwardly from $\mathcal{L}_{5}=-\phi G^{\mu \nu} \nabla_{\mu} \nabla_{\nu} \phi$, but that interaction can also be obtained equivalently from $\mathcal{L}_{4}=$ $X R+(\square \phi)^{2}-\left(\nabla_{\mu} \nabla_{\nu} \phi\right)^{2}$. We choose to employ the latter expression for new Higgs inflation. Hereafter, we write $g_{4}=g$.

The four remedies of Higgs inflation proposed so far can be reproduced by adding the extra term $\Delta \mathcal{L}$ to the standard Lagrangian, $M_{\mathrm{Pl}}^{2} R / 2+X-V(\phi)$, where $\Delta \mathcal{L}$ is given, respectively, by

$$
\Delta \mathcal{L}=\kappa \phi^{2 n} X \text { (running kinetic inflation), }
$$

$$
\Delta \mathcal{L}=\frac{\phi}{M^{4}} X \square \phi(\text { Higgs G-inflation), }
$$

$$
\Delta \mathcal{L}=-\frac{\xi}{2} \phi^{2} R \text { (nonminimal Higgs inflation), }
$$

and

$$
\Delta \mathcal{L}=\frac{1}{2 \mu^{2}}\left[X R+(\square \phi)^{2}-\left(\nabla_{\mu} \nabla_{\nu} \phi\right)^{2}\right]
$$

(new Higgs inflation).

Here $\kappa$ and $\xi$ are dimensionless constants, and $M$ and $\mu$ are parameters having dimension of mass. All of those apparently different models can be treated in a unified manner by taking

$$
\begin{gathered}
\mathcal{K}(\phi)=1+\kappa \phi^{2 n}, \\
h_{3}(\phi)=\frac{\phi}{M^{4}}, \\
g(\phi)=\frac{M_{\mathrm{Pl}}^{2}}{2}-\frac{\xi}{2} \phi^{2}, \\
h_{4}(\phi)=\frac{1}{2 \mu^{2}}, \\
h_{5}(\phi)=0 .
\end{gathered}
$$

It is then natural to imagine the case with $h_{5}(\phi) \neq 0,{ }^{2}$ which would lead to yet another successful Higgs inflation model that has not been explored before. We call it running Einstein inflation, since it is supported by the change of the coefficient of the Einstein tensor.

In the following analysis, we will consider all five possibilities of Higgs inflation on equal footing, by characterizing potential-driven inflation in terms of the five

\footnotetext{
${ }^{2}$ The simplest example of $h_{5}(\phi)$ for Higgs inflation is $h_{5}(\phi)=$ $\phi / \Lambda^{6}$, with $\Lambda$ being some cutoff scale. Note also that, in order to guarantee the gauge invariance of the Higgs doublet, the power of $\phi$ in $\mathcal{K}, g$, and $h_{4}$ must be even, while that in $h_{3}$ and $h_{5}$ must be odd.
} 
arbitrary functions of $\phi, \mathcal{K}, g, h_{3}, h_{4}, h_{5}$, besides the potential $V$.

\section{GENERAL SLOW-ROLL DYNAMICS OF POTENTIAL-DRIVEN INFLATION}

In order to investigate the general slow-roll dynamics of potential-driven inflation including the variants of Higgs inflation, we assume the following slow-roll conditions,

$$
\begin{aligned}
\epsilon & :=-\frac{\dot{H}}{H^{2}} \ll 1, \quad \eta:=-\frac{\ddot{\phi}}{H \dot{\phi}} \ll 1, \\
\delta & :=\frac{\dot{g}}{H g} \ll 1, \quad \alpha_{2}:=\frac{\dot{\mathcal{K}}}{H \mathcal{K}} \ll 1, \\
\alpha_{i} & :=\frac{\dot{h}_{i}}{H h_{i}} \ll 1 \quad(i=3,4,5) .
\end{aligned}
$$

We also assume that $\dot{\delta} / H \delta, \dot{\alpha}_{i} / H \alpha_{i} \ll 1(i=2,3,4,5)$. It is then found that

$$
J \simeq \mathcal{K} \dot{\phi}+3 h_{3} H \dot{\phi}^{2}+6 h_{4} H^{2} \dot{\phi}+3 h_{5} H^{3} \dot{\phi}^{2},
$$

and the slow-roll equation of motion for the inflaton is given by

$$
3 H J \simeq-V^{\prime}+12 H^{2} g^{\prime} .
$$

This is the generalized slow-roll equation of motion for $\phi$, where we can see how each term in Eq. (31) modifies the structure of the friction term. We can also see that the nonminimal coupling in $g$ changes effectively the slope of the potential.

We are considering potential-dominated inflation, so that $V \gg \mathcal{O}(\dot{\phi} J)$. Then, the gravitational field equations read

$$
\begin{gathered}
6 g H^{2} \simeq V, \\
-4 g \dot{H}+2 g^{\prime} \dot{\phi} H \simeq \dot{\phi} J .
\end{gathered}
$$

The second equation can be derived from Eqs. (32) and (33), or more directly from the space-space component of the gravitational field equations. From the Friedmann equation (33) one can see that $2 g$ may be regarded as an effective Planck mass squared. We should only consider the domain $g>0$ [13], which is always satisfied in the nonminimal Higgs inflation model since it adopts a large and negative $\xi$. Using Eq. (33), one can remove $H$ from the right-hand side of Eq. (32) to give

$$
3 H J \simeq-g^{2}\left(\frac{V}{g^{2}}\right)^{\prime}=:-U^{\prime}(\phi) .
$$

The effective potential $U$ coincides with that introduced in Refs. [26] to derive the slow-roll conditions in the Jordan frame.

Let us define

$$
u(\phi):=\mathcal{K}+\frac{h_{4} V}{g}, \quad v(\phi):=h_{3}+\frac{h_{5} V}{6 g} .
$$

Note that $u$ and $v$ are functions of $\phi$ only and are determined completely through the functions in the Lagrangian. Among the six functions of $\phi$ in the Lagrangian, the above particular combinations $u(\phi)$ and $v(\phi)$ are crucial for the slow-roll dynamics and the spectra of primordial fluctuations. In terms of $u$ and $v, J$ can be written as

$$
J=u \dot{\phi}+6 H X v .
$$

Plugging this expression into Eq. (35) and solving for $\dot{\phi}$, we get

$$
3 H \dot{\phi} \simeq \frac{1}{2 v}\left(-u+\sqrt{u^{2}-4 U^{\prime} v}\right) .
$$

Comparing this with the original equation (35), we find

$$
\frac{J}{\dot{\phi}} \simeq \frac{1}{2}\left(u+\sqrt{u^{2}-4 U^{\prime} v}\right)=: W(\phi) .
$$

We require $u^{2}-4 U^{\prime} v>0$ so that Eqs. (38) and (39) make sense. In addition, it may be reasonable to assume that $u>0$. We then have $0<u / W<2$. The consequences of this inequality will be discussed further in relation to the stability against linear perturbations in the next section.

Combining Eqs. (33) and (38), we arrive at

$$
\frac{d \phi}{d \mathcal{N}}=\frac{\dot{\phi}}{H} \simeq-2 \frac{g U^{\prime}}{V W},
$$

where $\mathcal{N}:=\ln a$ is the number of $e$-folds. Note that the right-hand side is expressed solely in terms of $\phi$ and reduces to $-M_{\mathrm{Pl}}^{2} V^{\prime} / V$ in the case of the standard canonical field. In general slow-roll inflation, the effective potential slope $2 g U^{\prime} / V W$ governs the motion of $\phi$ rather than the "bare" one $M_{\mathrm{Pl}}^{2} V^{\prime} / V$. For instance, slow roll of $\phi$ is possible even in a steep potential if $W \gg 1$. Equation (40) can be used to evaluate the number of $e$-folds until the end of inflation.

Using Eq. (40), each slow-roll parameter can be expressed in terms of the $\phi$-dependent functions as

$$
\begin{aligned}
\delta & \simeq-2 \frac{g^{\prime} U^{\prime}}{W V}, \\
\epsilon & \simeq \frac{g}{W}\left(\frac{U^{\prime}}{V}\right)^{2}-\frac{\delta}{2}, \\
\frac{j}{H J} & \simeq-2 \frac{g}{W} \frac{U^{\prime \prime}}{V}+\epsilon, \\
\alpha_{i} & \simeq-2 \frac{g U^{\prime}}{V W} \frac{h_{i}^{\prime}}{h_{i}} .
\end{aligned}
$$

Note that Eq. (42), together with $g>0$ and $W>0$, ensures

$$
\epsilon+\delta / 2>0
$$

The ratio $\dot{\phi} J / V$ can be expressed in terms of the slowroll parameters as 


$$
\frac{\dot{\phi} J}{V} \simeq \frac{2}{3} \epsilon+\frac{1}{3} \delta \ll 1 .
$$

From this, the initial assumption that the potential is dominant in the Friedmann equation is found to be consistent.

It is instructive here to demonstrate the extreme case where only $h_{5}$ is nontrivial, corresponding to the running Einstein inflation model we are proposing in this paper. In this case $U^{\prime}=V^{\prime}, u=1$, and $v=h_{5}(\phi) V(\phi) / 3 M_{\mathrm{Pl}}^{2}$. Noting that $\epsilon=\epsilon_{\text {std }} / W$, where $\epsilon_{\text {std }}:=\left(M_{\mathrm{Pl}}^{2} / 2\right)\left(V^{\prime} / V\right)^{2}$ is the standard slow-roll parameter defined in terms of the potential, we see that inflation proceeds even with a steep potential provided $W \gg 1$. This occurs in the domain where $\left|h_{5} V^{\prime} V / M_{\mathrm{Pl}}^{2}\right| \gg 1$ is satisfied.

\section{COSMOLOGICAL PERTURBATIONS IN GENERALIZED HIGGS INFLATION}

In this section, we study cosmological perturbations in generalized Higgs inflation and present useful formulas for the spectra of tensor and scalar perturbations. A generic formulation of cosmological perturbations in the most general single-field inflation model was already given in Ref. [21]. For completeness, we begin with duplicating the general formulas, and then illustrate how they can be applied to the potential-driven models.

\section{A. Generic formulation for linear perturbations}

It is convenient to write the perturbed metric in the Arnowitt-Deser-Misner form as

$$
d s^{2}=-N^{2} d t^{2}+\gamma_{i j}\left(d x^{i}+N^{i} d t\right)\left(d x^{j}+N^{j} d t\right),
$$

where

$$
\begin{aligned}
N & =1+\delta n, \quad N_{i}=\partial_{i} \chi, \\
\gamma_{i j} & =a^{2}(t) e^{2 \zeta}\left(\delta_{i j}+h_{i j}+\frac{1}{2} h_{i k} h_{k j}\right) .
\end{aligned}
$$

Here, $\delta n, \chi$, and $\zeta$ are scalar perturbations and $h_{i j}$ is a tensor perturbation satisfying $h_{i i}=0=h_{i j, j}$. We choose the unitary gauge in which $\phi(t, \mathbf{x})=\phi(t)$. Substituting the metric to the action and expanding it to second order in perturbations, we obtain the quadratic actions for the tensor and scalar perturbations. For the scalar perturbations, one may use the constraint equations to remove $\delta n$ and $\chi$ to get the quadratic action in terms of the single variable $\zeta$.

The quadratic action for the tensor perturbations is given by

$$
S_{T}^{(2)}=\frac{1}{8} \int d t d^{3} x a^{3}\left[G_{T} \dot{h}_{i j}^{2}-\frac{\mathcal{F}_{T}}{a^{2}}\left(\vec{\nabla} h_{i j}\right)^{2}\right],
$$

where

$$
\begin{gathered}
\mathcal{F}_{T}:=2\left[G_{4}-X\left(\ddot{\phi} G_{5 X}+G_{5 \phi}\right)\right], \\
G_{T}:=2\left[G_{4}-2 X G_{4 X}-X\left(H \dot{\phi} G_{5 X}-G_{5 \phi}\right)\right] .
\end{gathered}
$$

The squared sound speed is given by $c_{T}^{2}=\mathcal{F}_{T} / \mathcal{G}_{T}$. It is manifest from the action (49) that ghost and gradient instabilities are avoided provided that

$$
\mathcal{F}_{T}>0, \quad G_{T}>0 .
$$

Following the standard quantization procedure, the power spectrum of the primordial tensor perturbations is found to be

$$
\mathcal{P}_{T}=\left.8 \gamma_{T} \frac{\mathcal{G}_{T}^{1 / 2}}{\mathcal{F}_{T}^{3 / 2}} \frac{H^{2}}{4 \pi^{2}}\right|_{\text {sound horizon exit }},
$$

where $\quad \gamma_{T}=2^{2 \nu_{T}-3}\left|\Gamma\left(\nu_{T}\right) / \Gamma(3 / 2)\right|^{2}\left(1-\epsilon-f_{T} / 2+\right.$ $\left.g_{T} / 2\right)$. We emphasize that the power spectrum is evaluated at sound horizon exit, because the propagation speed of the tensor mode does not coincide with that of light in general. Here, we have assumed that $\epsilon:=-\dot{H} / H^{2} \simeq$ const,

$$
f_{T}:=\frac{\dot{\mathcal{F}}_{T}}{H \mathcal{F}_{T}} \simeq \text { const, } \quad \text { and } \quad g_{T}:=\frac{\dot{\mathcal{G}}_{T}}{H \mathcal{G}_{T}} \simeq \text { const }
$$

and defined

$$
\nu_{T}:=\frac{3-\epsilon+g_{T}}{2-2 \epsilon-f_{T}+g_{T}} .
$$

The tensor spectral tilt is evaluated as

$$
n_{T}=3-2 \nu_{T} \text {. }
$$

On the other hand, the quadratic action for the scalar perturbations is given by

$$
S_{S}^{(2)}=\int d t d^{3} x a^{3}\left[G_{S} \dot{\zeta}^{2}-\frac{\mathcal{F}_{S}}{a^{2}}(\vec{\nabla} \zeta)^{2}\right],
$$

where

$$
\begin{gathered}
\mathcal{F}_{S}:=\frac{1}{a} \frac{d}{d t}\left(\frac{a}{\Theta} G_{T}^{2}\right)-\mathcal{F}_{T}, \\
G_{S}:=\frac{\Sigma}{\Theta^{2}} G_{T}^{2}+3 G_{T},
\end{gathered}
$$

and $\Sigma$ and $\Theta$ are defined as

$$
\begin{aligned}
\Sigma:= & X K_{X}+2 X^{2} K_{X X}+12 H \dot{\phi} X G_{3 X}+6 H \dot{\phi} X^{2} G_{3 X X}-2 X G_{3 \phi}-2 X^{2} G_{3 \phi X}-6 H^{2} G_{4}+6\left[H ^ { 2 } \left(7 X G_{4 X}\right.\right. \\
& \left.\left.+16 X^{2} G_{4 X X}+4 X^{3} G_{4 X X X}\right)-H \dot{\phi}\left(G_{4 \phi}+5 X G_{4 \phi X}+2 X^{2} G_{4 \phi X X}\right)\right]+30 H^{3} \dot{\phi} X G_{5 X}+26 H^{3} \dot{\phi} X^{2} G_{5 X X} \\
& +4 H^{3} \dot{\phi} X^{3} G_{5 X X X}-6 H^{2} X\left(6 G_{5 \phi}+9 X G_{5 \phi X}+2 X^{2} G_{5 \phi X X}\right),
\end{aligned}
$$




$$
\begin{aligned}
\Theta:= & -\dot{\phi} X G_{3 X}+2 H G_{4}-8 H X G_{4 X}-8 H X^{2} G_{4 X X} \\
& +\dot{\phi} G_{4 \phi}+2 X \dot{\phi} G_{4 \phi X}-H^{2} \dot{\phi}\left(5 X G_{5 X}\right. \\
& \left.+2 X^{2} G_{5 X X}\right)+2 H X\left(3 G_{5 \phi}+2 X G_{5 \phi X}\right) .
\end{aligned}
$$

The squared sound speed of the curvature perturbations is given by $c_{S}^{2}=\mathcal{F}_{S} / \mathcal{G}_{S}$, and ghost and gradient instabilities are avoided provided that the following conditions are satisfied:

$$
\mathcal{F}_{S}>0, \quad G_{S}>0
$$

As is the case of the tensor perturbations, the power spectrum of the scalar perturbations can be easily computed as

$$
\mathcal{P}_{\zeta}=\left.\frac{\gamma_{S}}{2} \frac{G_{S}^{1 / 2}}{\mathcal{F}_{S}^{3 / 2}} \frac{H^{2}}{4 \pi^{2}}\right|_{\text {sound horizon exit }},
$$

where $\gamma_{S}=2^{2 \nu_{S}-3}\left|\Gamma\left(\nu_{S}\right) / \Gamma(3 / 2)\right|^{2}\left(1-\epsilon-f_{S} / 2+g_{S} / 2\right)$. Note that the (sound) horizon crossing time for $\zeta$ is different from that for $h_{i j}$, in general. We have assumed that $\epsilon \simeq$ const,

$$
f_{S}:=\frac{\dot{\mathcal{F}}_{S}}{H \mathcal{F}_{S}} \simeq \text { const, } \quad g_{S}:=\frac{\dot{\mathcal{G}}_{S}}{H \mathcal{G}_{S}} \simeq \text { const },
$$

and also define

$$
\nu_{S}:=\frac{3-\epsilon+g_{S}}{2-2 \epsilon-f_{S}+g_{S}} .
$$

The scalar spectral index is computed as

$$
n_{s}-1=3-2 \nu_{S} \text {. }
$$

\section{B. Primordial perturbations in generalized Higgs inflation}

Now we are in a position to derive concise and useful formulas for tensor and scalar fluctuations in generalized Higgs inflation. The four important functions in the quadratic actions are evaluated as

$$
\mathcal{F}_{T} \simeq G_{T} \simeq 2 g
$$

and

$$
\begin{aligned}
& \mathcal{F}_{S} \simeq \frac{X}{H^{2}} u+\frac{4 \dot{\phi} X}{H} v, \\
& \mathcal{G}_{S} \simeq \frac{X}{H^{2}} u+\frac{6 \dot{\phi} X}{H} v .
\end{aligned}
$$

It is convenient to rewrite $\mathcal{F}_{S}$ and $\mathcal{G}_{S}$ as

$$
\begin{aligned}
& \mathcal{F}_{S} \simeq \frac{g}{3}(2 \epsilon+\delta)\left(4-\frac{u}{W}\right), \\
& G_{S} \simeq g(2 \epsilon+\delta)\left(2-\frac{u}{W}\right),
\end{aligned}
$$

where we used Eqs. (33), (37), (39), and (46). We see that $\mathcal{F}_{T}, G_{T}>0$ since we are assuming that the effective Planck mass squared $g$ is positive. It should be noted that $\mathcal{F}_{S}>0$ and $G_{S}>0$ are also guaranteed by the inequalities $\epsilon+\delta / 2>0$ and $u / W<2$ which we discussed in the previous section. The sound speed squared is given by

$$
c_{s}^{2}=\frac{4-u / W}{3(2-u / W)} .
$$

We see that $2 / 3 \leq c_{s}^{2}<\infty$, though the superluminal propagation leads to the absence of the Lorentz invariant UV completion [27].

The tensor power spectrum is simply given by

$$
\mathcal{P}_{T} \simeq \frac{H^{2}}{\pi^{2} g} \simeq \frac{V}{6 \pi^{2} g^{2}}
$$

and its tilt is

$$
n_{T} \simeq-2 \epsilon-\delta .
$$

From (45) we find it is always negative in the potential-driven models under consideration, although the blue tensor spectrum is possible in kinetically driven $G$-inflation [6].

The power spectrum of the curvature perturbations is expressed as

$$
\mathcal{P}_{\zeta}=\frac{\sqrt{3}}{16 \pi^{2}} \frac{V}{g^{2}(2 \epsilon+\delta)} \frac{(2-u / W)^{1 / 2}}{(4-u / W)^{3 / 2}},
$$

and the spectral index is

$$
\begin{aligned}
n_{s}-1 \simeq & -4 \epsilon+\eta-\frac{j}{H J}+2 \frac{g U^{\prime}}{V W}\left[\frac{1}{2} \frac{(u / W)^{\prime}}{(2-u / W)}\right. \\
& \left.-\frac{3}{2} \frac{(u / W)^{\prime}}{(4-u / W)}\right] .
\end{aligned}
$$

Thus, the tensor-to-scalar ratio is given by

$$
r=-\frac{8}{3 \sqrt{3}} \frac{(4-u / W)^{3 / 2}}{(2-u / W)^{1 / 2}} n_{T}=-\frac{8}{\sqrt{3}}(4-u / W)^{1 / 2} c_{s} n_{T} .
$$

It is interesting to note that the tensor-to-scalar ratio is enhanced if the inflaton trajectory satisfies $u / W \approx 2$, i.e., $u^{2} \approx 4 U^{\prime} v$.

Let us then consider two extreme cases where

$$
J \simeq u \dot{\phi} \text { and } J \simeq 6 H X v .
$$

The former corresponds to $u \simeq W$, which is the case in running kinetic inflation and new Higgs inflation, and the latter to $u \ll W$, which is the case in Higgs $G$-inflation and running Einstein inflation. In both limiting cases we have $\mathcal{F}_{S}, G_{S} \propto\left(\dot{\phi} / H^{2}\right) J$, so that

$$
n_{s}-1 \simeq-4 \epsilon+\eta-\frac{\dot{J}}{H J} .
$$


If $J \simeq u \dot{\phi}$, the power spectrum is simplified to

$$
\mathcal{P}_{\zeta} \simeq \frac{1}{48 \pi^{2} g^{2}} \frac{V}{2 \epsilon+\delta} .
$$

In this case $\eta$ and $\dot{J} / H J$ are related via

$$
\eta \simeq-\frac{2 g U^{\prime}}{V W} \frac{u^{\prime}}{u}-\frac{\dot{J}}{H J} .
$$

Using this relation and Eq. (43), one can eliminate $\eta$ in Eq. (79) to express $n_{s}-1$ in terms of the $\phi$-dependent functions only. The consistency relation is nothing but the standard one:

$$
r \simeq-8 n_{T}
$$

On the other hand, if $J \simeq 6 H X v$ then the power spectrum reduces to

$$
\mathcal{P}_{\zeta} \simeq \frac{\sqrt{6}}{128 \pi^{2} g^{2}} \frac{V}{2 \epsilon+\delta} .
$$

In this case $\eta$ and $\dot{J} / H J$ are related via

$$
\eta \simeq-\frac{\epsilon}{2}-\frac{g U^{\prime}}{V W} \frac{v^{\prime}}{v}-\frac{1}{2} \frac{j}{H J},
$$

which allows us to write $n_{s}-1$ in terms of the $\phi$-dependent functions only. The consistency relation is given by the nonstandard one:

$$
r \simeq-\frac{32 \sqrt{6}}{9} n_{T}
$$

\section{Non-Gaussianity}

As with conventional potential-driven inflation models we expect small non-Gaussianity in the models at hand. It is explicitly computed in the Appendix. In the limit $u \gg$ $H \dot{\phi} v$, it turns out that the equilateral $f_{\mathrm{NL}}$ is slow-roll suppressed. In the opposite limit, $u \ll H \dot{\phi} v$, we find that the leading contribution is independent of the slow-roll parameters:

$$
f_{\mathrm{NL}} \simeq \frac{235}{3888} .
$$

However, in the special case $u / W \approx 2$ we have $c_{s}^{2} \gg 1$. In this case $f_{\mathrm{NL}}$ can be as large as

$$
f_{\mathrm{NL}} \approx \frac{5}{81} c_{s}^{2} \gg 1 \text {. }
$$

This happens if $u \approx-6 H \dot{\phi} v$.

\section{DISCUSSION}

We have presented a unified treatment of Higgs inflation models in the context of the most general single-field inflation model with second-order equations of motion, the generalized $G$-inflation, in which all existing Higgs inflation models can be accommodated. This unified approach also enabled us to find yet another class of Higgs inflation models, running Einstein inflation. Including this newly proposed model, we have studied five Higgs inflation models on the same footing. Formulas for primordial fluctuations of the generalized Higgs inflation were given, which would be quite useful to discuss and discriminate the model from observations and experiments in the near future such as the LHC and Planck satellite.

Although our analysis is applicable to a wide class of potential-driven inflation models besides the SM Higgs inflation, as for the relevance to the latter, it is important to analyze the stability of the theory at the energy scale of inflation. In fact, according to [28], for the mass range of the SM Higgs particle favored by the recent LHC result $[1,2]$, the parameter region where the Higgs quartic coupling is positive and stable up to the inflationary scale is disfavored, ${ }^{3}$ which might make all the Higgs inflation models difficult or even impossible. However, as already pointed out by the authors of Ref. [28], there are still theoretical uncertainties on the beta functions and experimental errors on the top and the Higgs masses, which make the conclusion indecisive. In addition, the presence of higher order derivatives and the coupling to the Einstein tensor may alter the results in the context of generalized Higgs inflation. Therefore, proper analysis must be performed in the framework of generalized $G$-inflation, which may well improve the situation. ${ }^{4}$ We plan to study this issue by the time the discovery of the Higgs particle is confirmed and its mass is fixed. After completion of this study, we can answer the question of whether inflation can be explained within the SM or not. ${ }^{5}$

\section{ACKNOWLEDGMENTS}

We would like to thank Matt Lake for a useful comment. This work was partially supported by JSPS Grant-in-Aid for Research Activity Start-up No. 22840011 (T. K.); Grants-in-Aid for Scientific Research No. 23740195 (T. T.), No. 21740187 (M. Y.), and No. 23340058 (J.Y.); and the Grant-in-Aid for Scientific Research on Innovative Areas No. 21111006 (J. Y.).

\section{APPENDIX: BISPECTRUM}

In this appendix, we present the explicit formula for the bispectrum in general potential-driven slow-roll inflation. The bispectrum of the curvature perturbation is defined as

\footnotetext{
${ }^{3}$ The possibility that the Higgs quartic coupling and its beta function vanish at the Planck scale has also been discussed in Ref. [29].

${ }^{4}$ It is known that the introduction of additional degrees of freedom relaxes the constraints [30].

${ }^{5}$ Possible extensions of the SM accommodating Higgs inflationary scenarios are discussed in Ref. [31].
} 


$$
\left\langle\zeta_{\mathbf{k}_{1}} \zeta_{\mathbf{k}_{2}} \zeta_{\mathbf{k}_{3}}\right\rangle=(2 \pi)^{3} \delta\left(\mathbf{k}_{1}+\mathbf{k}_{2}+\mathbf{k}_{3}\right) B_{\zeta}\left(k_{1}, k_{2}, k_{3}\right) .
$$

Following the result of Refs. [32-34], we find

$$
\begin{aligned}
B_{\zeta}= & \frac{(2 \pi)^{4} \mathcal{P}_{\zeta}^{2}}{4 k_{1}^{3} k_{2}^{3} k_{3}^{3}}\left[6 \mathcal{C}_{1} \frac{\left(k_{1} k_{2} k_{3}\right)^{2}}{K^{3}}+\frac{\mathcal{C}_{2}}{K}\left(2 \sum_{i>j} k_{i}^{2} k_{j}^{2}-\frac{1}{K} \sum_{i \neq j} k_{i}^{2} k_{j}^{3}\right)\right. \\
& +\mathcal{C}_{3}\left(\sum_{i} k_{i}^{3}+\frac{4}{K} \sum_{i>j} k_{i}^{2} k_{j}^{2}-\frac{2}{K^{2}} \sum_{i \neq j} k_{i}^{2} k_{j}^{3}\right) \\
& \left.+\frac{\mathcal{C}_{4}}{K}\left(\sum_{i} k_{i}^{4}-2 \sum_{i>j} k_{i}^{2} k_{j}^{2}\right)\left(1+\frac{1}{K^{2}} \sum_{i>j} k_{i} k_{j}+\frac{3 k_{1} k_{2} k_{3}}{K^{3}}\right)\right],
\end{aligned}
$$

where $K:=k_{1}+k_{2}+k_{3}$, each coefficient $\mathcal{C}_{i}$ is given by

$$
\begin{aligned}
\mathcal{C}_{1}=\frac{1}{c_{s}^{2}}-1+\frac{2\left(2-c_{s}^{2}\right)}{\mathcal{F}_{S}} \frac{\dot{\phi} X}{H} v \\
\simeq \frac{1}{c_{s}^{2}}-1+2\left(2-c_{s}^{2}\right) \frac{1-u / W}{4-u / W}, \\
\mathcal{C}_{2}=3\left(1-\frac{1}{c_{s}^{2}}\right), \\
\mathcal{C}_{3}=\frac{1}{2}\left(\frac{1}{c_{s}^{2}}-1\right),
\end{aligned}
$$

$$
\mathcal{C}_{4}=-\frac{1}{c_{s}^{2} \mathcal{F}_{S}} \frac{\dot{\phi} X}{H} v \simeq-\frac{1}{c_{s}^{2}} \frac{1-u / W}{4-u / W}
$$

and we have neglected the slow-roll suppressed contributions. Here, we have also used the relations

$$
\begin{gathered}
\mathcal{F}_{S}=2 g \epsilon+g \delta+\frac{\dot{\phi} X}{H} v, \\
\frac{\dot{\phi} X}{H} v \simeq \frac{g}{3}(2 \epsilon+\delta)\left(1-\frac{u}{W}\right) .
\end{gathered}
$$

It is found that

$$
\mathcal{C}_{1}, \mathcal{C}_{2}, \mathcal{C}_{3}, \mathcal{C}_{4} \lesssim 1
$$

for $c_{s}^{2}=\mathcal{O}(1)$. However, one of the coefficients can be as large as $\mathcal{C}_{1} \sim c_{s}^{2} \gg 1$ for $u / W \approx 2$.

Taking the equilateral configuration, $k_{1}=k_{2}=k_{3}$, the nonlinearity parameter $f_{\mathrm{NL}}$ is given by

$$
\begin{aligned}
f_{\mathrm{NL}} & =\frac{5}{81}\left(\mathcal{C}_{1}+6 \mathcal{C}_{2}+\frac{51}{2} \mathcal{C}_{3}-\frac{13}{2} \mathcal{C}_{4}\right) \\
& \simeq \frac{5}{243} \frac{(1-u / W)^{2}(99-43 u / W)}{(4-u / W)^{2}(2-u / W)} .
\end{aligned}
$$

[1] ATLAS Collaboration, Phys. Lett. B 710, 49 (2012).

[2] S. Chatrchyan et al. (CMS Collaboration), Phys. Lett. B 710, 26 (2012).

[3] K. Sato, Mon. Not. R. Astron. Soc. 195, 467 (1981); A. H. Guth, Phys. Rev. D 23, 347 (1981); A. D. Linde, Phys. Lett. 108B, 389 (1982); A. Albrecht and P. J. Steinhardt, Phys. Rev. Lett. 48, 1220 (1982).

[4] A. D. Linde, Phys. Lett. 129B, 177 (1983).

[5] C. Armendariz-Picon, T. Damour, and V.F. Mukhanov, Phys. Lett. B 458, 209 (1999).

[6] T. Kobayashi, M. Yamaguchi, and J. Yokoyama, Phys. Rev. Lett. 105, 231302 (2010).

[7] A. A. Starobinsky, Phys. Lett. 91B, 99 (1980).

[8] J.R. Ellis, N. Kaloper, K. A. Olive, and J. Yokoyama, Phys. Rev. D 59, 103503 (1999).

[9] V.F. Mukhanov and G. V. Chibisov, Pis'ma Zh. Eksp. Teor. Fiz. 33, 549 (1981) [JETP Lett. 33, 532 (1981)].S. W. Hawking, Phys. Lett. 115B, 295 (1982); A. A. Starobinsky, Phys. Lett. 117B, 175 (1982); A. H. Guth and S-Y. Pi, Phys. Rev. Lett. 49, 1110 (1982).

[10] D. S. Salopek, Phys. Rev. Lett. 69, 3602 (1992).

[11] M. B. Einhorn and D. R. T. Jones, J. High Energy Phys. 03 (2010) 026; S. Ferrara, R. Kallosh, A. Linde, A. Marrani, and A. Van Proeyen, Phys. Rev. D 82, 045003 (2010); H. M. Lee, J. Cosmol. Astropart. Phys. 08 (2010) 003; S. Ferrara, R. Kallosh, A. Linde, A. Marrani, and A. Van
Proeyen, Phys. Rev. D 83, 025008 (2011); A. Chatterjee and A. Mazumdar, J. Cosmol. Astropart. Phys. 09 (2011) 009; M. Arai, S. Kawai, and N. Okada, Phys. Rev. D 84, 123515 (2011); C. Pallis and N. Toumbas, J. Cosmol. Astropart. Phys. 12 (2011) 002; M. Arai, S. Kawai, and N. Okada, arXiv:1112.2391.

[12] B. L. Spokoiny, Phys. Lett. 147B, 39 (1984).

[13] T. Futamase and K.-i. Maeda, Phys. Rev. D 39, 399 (1989).

[14] D. S. Salopek, J. R. Bond, and J. M. Bardeen, Phys. Rev. D 40, 1753 (1989); R. Fakir and W. G. Unruh, Phys. Rev. D 41, 1783 (1990); D. I. Kaiser, Phys. Rev. D 52, 4295 (1995); J. L. Cervantes-Cota and H. Dehnen, Nucl. Phys. B442, 391 (1995); E. Komatsu and T. Futamase, Phys. Rev. D 59, 064029 (1999); S. Tsujikawa and B. Gumjudpai, Phys. Rev. D 69, 123523 (2004); Y. Watanabe and E. Komatsu, Phys. Rev. D 75, 061301 (2007); F. L. Bezrukov and M. Shaposhnikov, Phys. Lett. B 659, 703 (2008); A. O. Barvinsky, A. Y. Kamenshchik, and A. A. Starobinsky, J. Cosmol. Astropart. Phys. 11 (2008) 021; F. Bezrukov, D. Gorbunov, and M. Shaposhnikov, J. Cosmol. Astropart. Phys. 06 (2009) 029; J. Garcia-Bellido, D. G. Figueroa, and J. Rubio, Phys. Rev. D 79, 063531 (2009); A. De Simone, M. P. Hertzberg, and F. Wilczek, Phys. Lett. B 678, 1 (2009); F. L. Bezrukov, A. Magnin, and M. Shaposhnikov, Phys. 
Lett. B 675, 88 (2009); F. Bezrukov and M. Shaposhnikov, J. High Energy Phys. 07 (2009) 089; A. O. Barvinsky, A. Y. Kamenshchik, C. Kiefer, A. A. Starobinsky, and C. Steinwachs, J. Cosmol. Astropart. Phys. 12 (2009) 003; A. O. Barvinsky, A. Y. Kamenshchik, C. Kiefer, A. A. Starobinsky, and C. F. Steinwachs, arXiv:0910.1041; F. Bezrukov, A. Magnin, M. Shaposhnikov, and S. Sibiryakov, J. High Energy Phys. 01 (2011) 016; Y. Watanabe, Phys. Rev. D 83, 043511 (2011); I. Masina and A. Notari, arXiv:1112.2659 [Phys. Rev. D (to be published)]; Phys. Rev. Lett. 108, 191302 (2012); arXiv:1204.4155.

[15] C. Germani and A. Kehagias, Phys. Rev. Lett. 105, 011302 (2010); J. Cosmol. Astropart. Phys. 05 (2010) 019; 06 (2010) E01; C. Germani and Y. Watanabe, J. Cosmol. Astropart. Phys. 07 (2011) 031; 07 (2011) A01.

[16] L. N. Granda, J. Cosmol. Astropart. Phys. 04 (2011) 016; L. N. Granda and W. Cardona, J. Cosmol. Astropart. Phys. 07 (2010) 021 .

[17] K. Nakayama and F. Takahashi, J. Cosmol. Astropart. Phys. 11 (2010) 009; 02 (2011) 010.

[18] K. Kamada, T. Kobayashi, M. Yamaguchi, and J. Yokoyama, Phys. Rev. D 83, 083515 (2011).

[19] A. Nicolis, R. Rattazzi, and E. Trincherini, Phys. Rev. D 79, 064036 (2009).

[20] C. Deffayet, G. Esposito-Farese, and A. Vikman, Phys. Rev. D 79, 084003 (2009); C. Deffayet, S. Deser, and G. Esposito-Farese, Phys. Rev. D 80, 064015 (2009).

[21] T. Kobayashi, M. Yamaguchi, and J. Yokoyama, Prog. Theor. Phys. 126, 511 (2011).

[22] L. A. Popa, J. Cosmol. Astropart. Phys. 10 (2011) 025 .
[23] R. Barate et al. (LEP Working Group for Higgs boson searches, ALEPH, DELPHI, L3, and OPAL Collaborations), Phys. Lett. B 565, 61 (2003).

[24] G. W. Horndeski, Int. J. Theor. Phys. 10, 363 (1974).

[25] C. Deffayet, X. Gao, D. A. Steer, and G. Zahariade, Phys. Rev. D 84, 064039 (2011).

[26] T. Chiba and M. Yamaguchi, J. Cosmol. Astropart. Phys. 10 (2008) 021; T. Chiba and M. Yamaguchi, J. Cosmol. Astropart. Phys. 01 (2009) 019.

[27] A. Adams, N. Arkani-Hamed, S. Dubovsky, A. Nicolis, and R. Rattazzi, J. High Energy Phys. 10 (2006) 014.

[28] J. Elias-Miro, J. R. Espinosa, G. F. Giudice, G. Isidori, A. Riotto, and A. Strumia, Phys. Lett. B 709, 222 (2012).

[29] D. L. Bennett, C. D. Froggatt, and H. B. Nielsen, Report No. NBI-HE-94-44; C. D. Froggatt and H. B. Nielsen, Phys. Lett. B 368, 96 (1996); C.D. Froggatt, H. B. Nielsen, and Y. Takanishi, Phys. Rev. D 64, 113014 (2001); M. Shaposhnikov and C. Wetterich, Phys. Lett. B 683, 196 (2010); M. Holthausen, K. S. Lim, and M. Lindner, J. High Energy Phys. 02 (2012) 037.

[30] O. Lebedev, arXiv:1203.0156; J. Elias-Miro, J.R. Espinosa, G. F. Giudice, H. M. Lee, and A. Strumia, arXiv:1203.0237.

[31] M. Shaposhnikov and D. Zenhausern, Phys. Lett. B 671, 187 (2009); M. Shaposhnikov and D. Zenhausern, Phys. Lett. B 671, 162 (2009); J. Garcia-Bellido, J. Rubio, M. Shaposhnikov, and D. Zenhausern, Phys. Rev. D 84, 123504 (2011).

[32] X. Gao and D. A. Steer, J. Cosmol. Astropart. Phys. 12 (2011) 019.

[33] A. De Felice and S. Tsujikawa, Phys. Rev. D 84, 083504 (2011).

[34] S. Renaux-Petel, J. Cosmol. Astropart. Phys. 02 (2012) 020 . 